\title{
The videolaryngoscope is less traumatic than the classic laryngoscope for a difficult airway in an obese patient
}

\author{
Ralph MaAssen ${ }^{1,2}$, Ruben LeE ${ }^{3}$, André van Zundert ${ }^{1,4}$, and Richard Cooper ${ }^{5}$ \\ ${ }^{1}$ Department of Anesthesiology, Intensive Care and Pain Therapy, Catharina Hospital—Brabant Medical School, Eindhoven, \\ The Netherlands \\ ${ }^{2}$ University of Maastricht, Maastricht, The Netherlands \\ ${ }^{3}$ Department of Biomechanical Engineering, 3ME, Delft University of Technology, Delft, The Netherlands \\ ${ }^{4}$ University of Ghent, Ghent, Belgium \\ ${ }^{5}$ Department of Anesthesiology, Toronto General Hospital, University of Toronto, Toronto, Canada
}

\begin{abstract}
This report describes the anesthetic management of an obese patient with a difficult airway and the merits of videolaryngoscopy, specifically in terms of the reduced risk of dental damage during intubation. A 49-year-old woman (body mass index; BMI, $36 \mathrm{~kg} \cdot \mathrm{m}^{-2}$ ), was scheduled to undergo an elective laparoscopic cholecystectomy because of cholelithiasis. Based upon the obesity of the patient and preoperative metrics (Mallampati grade IV; interdental distance of $2.9 \mathrm{~cm}$; thyromental distance, $5.5 \mathrm{~cm}$ ) a difficult airway was anticipated. Classic direct laryngoscopy using a Macintosh blade size IV failed, despite three intubation attempts-each resulting in a CormackLehane grade IV view. Intubation using a video-assisted Macintosh laryngoscope (V-Mac; Karl Storz, Tuttlingen, Germany) was successful upon the first attempt. The maximum force exerted on the patient's maxillary incisors was $61 \mathrm{~N}$ by direct laryngoscopy and $7.6 \mathrm{~N}$ using the indirect videolaryngoscope, both using a Macintosh blade.
\end{abstract}

Key words General anesthesia · Intubation · Videolaryngoscopy $\cdot$ Difficult airway $\cdot$ Force measurement

\section{Introduction}

The recent introduction of laryngoscopes incorporating optics into the blade has improved glottic visualization [1-5]. However, it is not yet clear that this reduces airway trauma or reduces intubation times. Common tests, designed to predict difficult laryngoscopy, are of uncertain relevance when videolaryngoscopy is employed [6-8].

In this report, we review a case that demonstrates the value of a videolaryngoscope for the management of a difficult airway in an obese patient, using the V-Mac

Address correspondence to: A. van Zundert, Department of Anesthesiology, Catharina Hospital, Michelangelolaan 2, NL5623 EJ Eindhoven, The Netherlands

Received: December 29, 2008 / Accepted: April 17, 2009
Storz videolaryngoscope (Karl Storz, Tuttlingen, Germany). We measured forces applied to the maxillary incisors during direct laryngoscopy using a Macintosh blade size IV and the videolaryngoscope. This challenging case was an extraordinary inclusion in a recent study [9] measuring forces on the maxillary incisors during intubation, in which the attending anesthesiologist was blinded to the applied forces. Institutional Medical Ethics Committee approval and the patient's written informed consent were obtained.

\section{Case report}

A 49-year-old woman (height, $1.69 \mathrm{~m}$; weight, $104 \mathrm{~kg}$; body mass index [BMI], $36.4 \mathrm{~kg} \cdot \mathrm{m}^{-2}$ ) presented for elective laparoscopic cholecystectomy. She was classified as American Society of Anesthesiologists (ASA) II. Airway characteristics included a reduced interdental distance $(2.9 \mathrm{~cm})$, full dentition, reduced oropharyngeal view (Mallampati grade IV), reduced thyromental distance $(5.5 \mathrm{~cm})$, and normal neck movement. Both a poor glottic view and difficult intubation were anticipated.

Patient positioning was duly adjusted carefully into the ramped position prior to the induction of anesthesia, using pillows and blankets to bring the patient's sternal notch and the external auditory meatus into an imaginary horizontal line [10,11]. Direct laryngoscopy using a Macintosh blade size IV was initially selected for use with this patient. According to the study protocol, if intubation by direct laryngoscopy could not be achieved within $90 \mathrm{~s}$, a backward, upward and rightsided pressure on the thyroid and cricoid cartilages (BURP) maneuver $[12,13]$ was to be employed. If the BURP maneuver failed to produce laryngeal exposure, the protocol called for the use of a videolaryngoscope.

We measured the forces on the laryngoscope blades with Flexiforce sensors (A201-25; Tekscan, South 


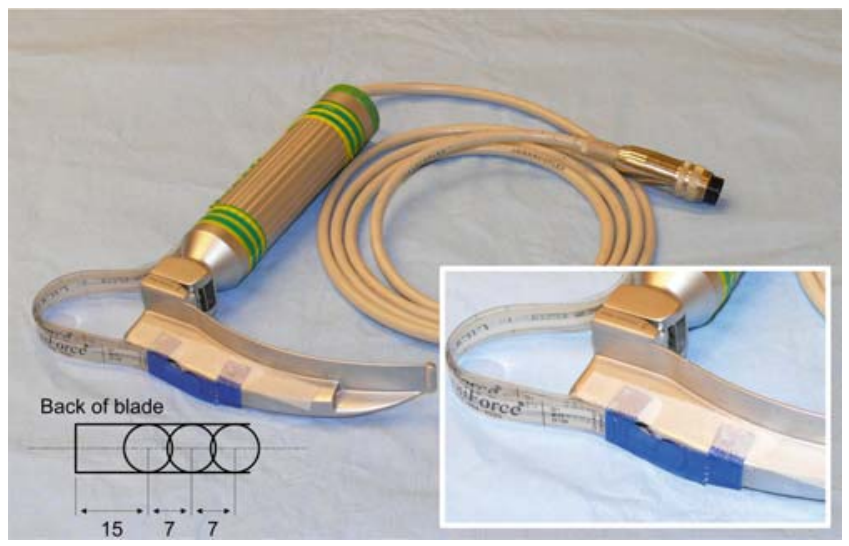

Fig. 1. Macintosh laryngoscope with sensors attached; detail of the placement of the Flexiforce (Tekscan, Boston, MA, USA) sensors used. The schematic shows the location of the sensors toward the rear of the laryngoscope blade

Boston, MA, USA) attached to the blade of the laryngoscope at the area of contact with the maxillary teeth [9] (Fig. 1). Three sensors were mounted along the length of the blade to sufficiently cover the surface likely to contact the teeth, given that the contact point could vary for the patient. Calibration was performed by applying a known mass ( 1 to $12 \mathrm{~kg}$, in steps of $1 \mathrm{~kg}$ ), using a flat-headed screwdriver (as geometrical approximation of the contact with the teeth), to the sensors mounted upon the blade. The sensors were invariant to the contact point of the applied load. Data acquisition was achieved with a National Instruments DAQ6009 (National Instruments, Austin, TX, USA) card at $500 \mathrm{~Hz}$, using Labview 7.0 (National Instruments) on a laptop computer (Hewlett Packard, Palo Alto, CA, USA). Peak forces were subsequently noted for the laryngoscopy of the patient.

After preoxygenation for 3 min, induction of anesthesia was carried out with intravenous $1 \mu \mathrm{g} \cdot \mathrm{kg}^{-1}$ fentanyl, $3 \mathrm{mg} \cdot \mathrm{kg}^{-1}$ propofol, and rocuronium $0.7 \mathrm{mg} \cdot \mathrm{kg}^{-1}$. The lungs were manually inflated via a facemask, using sevoflurane in oxygen. The laryngoscope was inserted approximately $2 \mathrm{~min}$ after completion of induction. Three intubation attempts with direct laryngoscopy failed to secure the airway, and despite external laryngeal manipulation and BURP, all revealed a CormackLehane (C\&L) grade IV view. The anesthesiologist then decided to use the V-Mac Storz videolaryngoscope (Karl Storz, Tuttlingen, Germany) [14] being trialled in the hospital. Videolaryngoscopy revealed a $C \& L$ grade I view and intubation was successful on the first attempt. The force measurements were made by an assistant, and the intubating anesthesiologist was blinded to these measurements. The maximum force on the maxillary teeth was $61.0 \mathrm{~N}$ using Macintosh direct laryngoscopy, and $7.6 \mathrm{~N}$ using indirect videolaryngoscopy (Fig. 2).
Forces during intubation of an obese patient with both classic and video laryngoscope

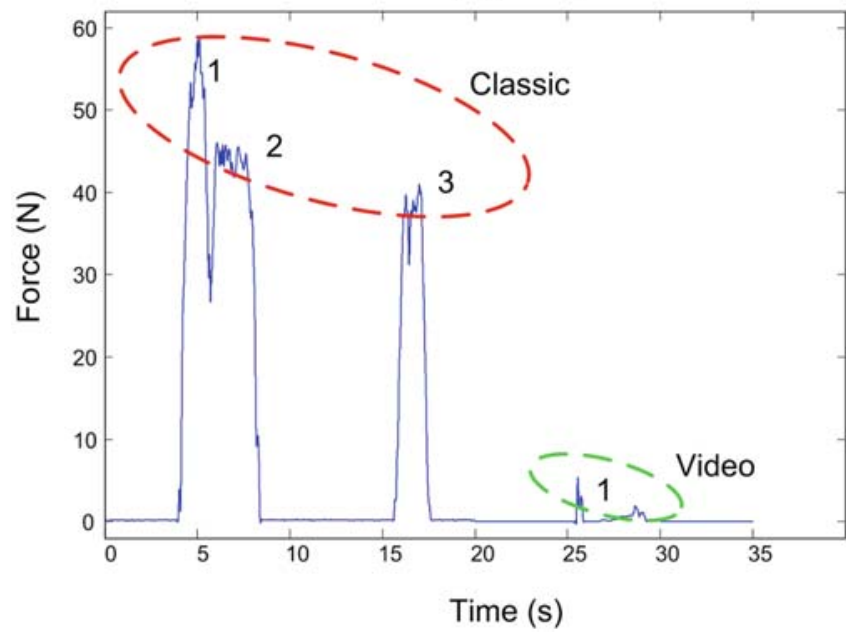

Fig. 2. Three attempts (indicated) at intubation with the classic (direct) laryngoscope show a high force applied to the maxillary incisors of the patient while trying to acquire a view of the glottis, whereas a much smaller force was applied with the (indirect) videolaryngoscope

Peripheral oxygen saturation during this procedure stayed at more than $95 \%$, due to intermittent manual bag ventilation with $100 \%$ oxygen, between the laryngoscopy attempts. At the end of the surgical procedure, following reversal of the neuromuscular blockade, the patient resumed spontaneous ventilation and was extubated, fully awake in an upright position. No complications were encountered.

\section{Discussion}

Numerous studies using a variety of devices have compared direct laryngoscopy and indirect videolaryngoscopy. All have shown that videolaryngoscopy consistently results in improved glottic visualization [1-5]. But it remains to be demonstrated that this ensures that intubation is either faster or less traumatic. To this end, measurement of the forces applied to the maxillary incisors could be an objective measurement of the intubation difficulty or the potential for dental injury $[15,16]$. Recently, our group found that, when using videoassisted laryngoscopes, the forces exerted by the intubating anesthesiologist on the patient's maxillary incisors were reduced compared with the forces exerted with the classic Macintosh laryngoscope [9]. However, it still has to be proved whether the same will be the case in patients with difficult airways.

We were able to make continuous registrations of the forces exerted on the maxillary teeth during the intubation process in the patient reported here. The forces 
measured in this case report were significantly reduced when using the Storz indirect video-assisted laryngoscope compared with direct laryngoscopy, both with a Macintosh blade. The attending anesthesiologist was unaware of the forces he was exerting. Additionally, the glottic view was considerably better and intubation was successful with the videolaryngoscope on the first pass. Videolaryngoscopy does not just display the same view on a monitor screen as that shown by convenventional direct laryngoscopy; the video captured by a videolaryngoscope is very different from that of conventional direct laryngoscopy. It not only offers visualization of the glottic entrance but also shows a distal viewpoint unachievable with direct classical techniques. The Storz videolaryngoscope is inserted in a way similar to that used for a classic laryngoscope blade, as both use a Macintosh blade, diverting the tongue to the midline, making room for the insertion of an endotracheal tube. The manufacturers of other videolaryngoscopes (GlideScope, Verathon, WA, USA and Mc.Grath Series 5, Aircraft Medical, Edinburgh, UK) advocate midline insertion and the use of a styletted endotracheal tube. Both the Airway Scope (Pentax-AWS system; AWS; Pentax, Tokyo, Japan) and the Airtraq (ATQ; Prodol Meditec, Vizcaya, Spain) are anatomically shaped rigid indirect videolaryngoscope intubation devices with an adjustable built-in monitor; both devices provide an indirect view of the glottis without requiring the laryngoscope's line of sight [17-19]. These devices use a tube channel in the right side of the blade which holds the endotracheal tube and directs it towards the vocal cords.

The application of the BURP technique and careful positioning of the patient had little influence on the laryngeal view $[20,21]$. Difficult airways pose a grave risk to the patient, especially in emergency situations. In a case such as the one described above (obese patient; Mallampati grade IV; C\&L, grade IV), it would often be necessary to enlist the help of additional devices and personnel. The excellent glottic view provided by the videolaryngoscope made such ancillary equipment unnecessary, and, as this case shows, reduced the need for excessive force on the laryngoscope.

A caveat remaining is that this case points to reduced forces applied to the maxillary incisors of a single patient, with a single videolaryngoscope; it is yet to be demonstrated this is also the case with different models of videolaryngoscopes. Although any contact with the maxillary teeth is undesirable, it is sometimes unavoidable when difficult laryngoscopies are encountered with classical direct laryngoscopy. Indeed, the incidence of accidental dental damage points to this phenomenon, which undoubtedly goes hand in hand with difficulty of intubation [22,23]. Perianesthetic dental injury (incidence varies from $0.04 \%$ to $12.08 \%$ ) is considered to be the most frequent (one third of total) anesthesia-related cause for insurance claims [22,24].

In planned studies we intend to more extensively investigate the performance of various types of videolaryngoscopes; specifically, the ease of intubation in the most challenging patients previously - those with high BMI and poor Mallampati grades.

\section{Conclusion}

The present case report shows that less force was needed to visualize the glottis entrance during laryngoscopy with Macintosh blades using indirect videolaryngoscopy compared with direct laryngoscopy. Dental damage may be reduced through better facilitated viewing of the glottis. Visualization and intubation were easy with the videolaryngoscope; especially in obese patients, the videolaryngoscope may provide an alternative intubation device and show better, less traumatic intubation conditions than the classic laryngoscope.

Acknowledgments. The authors sincerely thank Guy van Dael, Mike Roelofs, and Willem Binnendijk, from the Audiovisual Department, Catharina Hospital, Eindhoven, the Netherlands, for their help with the pictures.

\section{References}

1. Cooper RM. Use of a new videolaryngoscope (GlideScope) in the management of a difficult airway. Can J Anaesth. 2003;50: 611-3.

2. Cooper RM, Pacey JA, Bishop MJ, McCluskey SA. Early clinical experience with a new videolaryngoscope (GlideScope) in 728 patients. Can J Anaesth. 2005;52:191-8.

3. Kaplan MB, Hagberg CA, Ward DS, Brambrink A, Chhibber AK, Heidegger T, Lozada L, Ovassapian A, Parsons D, Ramsay J, Wilhelm W, Zwissler B, Gerig HJ, Hofstetter C, Karan S, Kreisler N, Pousman RM, Thierbach A, Wrobel M, Berci G. Comparison of direct and video-assisted views of the larynx during routine intubation. J Clin Anesth. 2006;18:357-62.

4. Kaplan MB, Ward D, Hagberg CA, Berci G, Hagiike M. Seeing is believing: the importance of video laryngoscopy in teaching and in managing the difficult airway. Surg Endosc. 2006;20 Suppl 2: S479-83.

5. Shippey B, Ray D, McKeown D. Case series: the McGrath videolaryngoscope-an initial clinical evaluation. Can J Anaesth. 2007;54:307-13.

6. Cormack RS, Lehane J. Difficult tracheal intubation in obstetrics. Anaesthesia. 1984;39:1105-11.

7. Lee A, Fan LT, Gin T, Karmakar MK, Ngan Kee WD. A systematic review (meta-analysis) of the accuracy of the Mallampati tests to predict the difficult airway. Anesth Analg. 2006;102:1867-78.

8. Mallampati SR, Gatt SP, Gugino LD, Desai LD, Waraksa B, Freiberger D, Liu PL. A clinical sign to predict difficult tracheal intubation: a prospective study. Can Anaesth Soc J. 1985;32: 429-34.

9. Lee RA, van Zundert AAJ, Maassen RLJG, Willems RJ, Beeke LP, Schaaper JN, van Dobbelsteen J, Wieringa PA. Forces applied to the maxillary incisors during video-assisted intubation. Anesth Analg. 2009;108:187-91. 
10. Brodsky JB, Lemmens HJM, Brock-Utne JG, Saidman LJ, Levitan R. Anesthetic considerations for bariatric surgery: proper positioning is important for laryngoscopy. Anesth Analg. 2003; 96:1841-2.

11. Rich JM. Use of an elevation pillow to produce the head-elevated laryngoscopy position for airway management in morbidly obese and large-framed patients. Anesth Analg. 2004;98:264-5.

12. Knill RL. Difficult laryngoscopy made easy with a "BURP". Can J Anesth. 1993;40:279-82.

13. Takahata O, Kubota M, Mamiya K, Akama Y, Nozaka T, Matsumoto H, Ogawa H. The efficacy of the "BURP" maneuver during a difficult laryngoscopy. Anesth Analg. 1997;84:419-21.

14. Van Zundert A, Stessel B, De Ruiter F, Giebelen D, Weber E. Video-assisted laryngoscopy: a useful adjunct in endotracheal intubation. Acta Anaesthesiol Belg. 2007;58:129-31.

15. Bucx MJ, Snijders CJ. Force, torque, and stress relaxation with direct laryngoscopy. Anesth Analg. 1996;83:1130-1.

16. Bucx MJ, van der Vegt MH, Snijders CJ, Stijnen T, Wesselink PR. Transverse forces exerted on the maxillary incisors during laryngoscopy. Can J Anaesth. 1996;43:665-71.

17. Suzuki A, Abe N, Sasakawa T, Kunisawa T, Takata O, Iwasaki H. Pentax-AWS (Airway Scope) and Airtraq: big difference between two similar devices. J. Anesth. 2008;22:191-2.
18. Hirabayashi Y, Seo N. Airway Scope: early clinical experience in 405 patients. J Anesth. 2008;22:81-5.

19. Suzuki A, Terao M, Aizawa K, Sasakawa T, Henderson JJ, Iwasaki H. Pentax-AWS Airway Scope as an alternative for awake flexible fiberoptic intubation of a morbidly obese patient in the semi-sitting position. J Anesth. 2009;23:162-3.

20. Snider DD, Clarke D, Finucane BT. The "BURP" maneuver worsens the glottic view when applied in combination with cricoid pressure. Can J Anaesth. 2005;52:100-4.

21. Takahata O, Kubota M, Mamiya K, Akama Y, Nozaka T, Matsumoto H, Ogawa H. The efficacy of the "BURP" maneuver during a difficult laryngoscopy. Anesth Analg. 1997;84:419-21.

22. Givol N, Gershtansky Y, Halamish-Shani T, Taicher S, Perel A, Segal E. Perianesthetic dental injuries: analysis of incident reports. J Clin Anesth. 2004;16:173-6.

23. Warner ME, Benenfeld SM, Warner MA, Schroeder DR, Maxon PM. Perianesthetic dental injuries: frequency, outcomes, and risk factors. Anesthesiology. 1999;90:1302-5.

24. Newland MC, Ellis SJ, Peters KR, Simonson JA, Durham TM, Ullrich FA, Tinker JH. Dental injury associated with anesthesia: a report of 161687 anesthetics given over 14 years. J Clin Anesth. 2007;19:339-45. 